\title{
Nanomoulding of transparent zinc oxide electrodes for efficient light trapping in solar cells
}

\author{
Corsin Battaglia*, Jordi Escarré, Karin Söderström, Mathieu Charrière, Matthieu Despeisse, \\ Franz-Josef Haug and Christophe Ballif
}

Nanopatterning has gained tremendous importance in the field of photovoltaics, as absorption of sunlight in solar cells can be enhanced drastically by proper engineering of photonic nanostructures $^{1-8}$. However, despite intensive efforts, neither the ideal surface morphology nor the ideal scattering characteristics for optimum light trapping have been identified. Experimentally, a method capable of implementing arbitrarily designed surface morphologies directly into functional devices is desirable. Here, we establish a nanomoulding process that provides exactly such a platform, enabling precise, large-area, nanoscale patterning of functional zinc oxide films at low cost. We illustrate the application of nanomoulded zinc oxide films as transparent front electrodes in amorphous silicon solar cells, demonstrating excellent initial conversion efficiencies of $10.1 \%$. In the quest to find the most efficient light-harvesting scheme, we anticipate that nanomoulding will catalyse the development and integration of exciting new nanophotonic structures.

Zinc oxide $(\mathrm{ZnO})$ is currently one of the key functional materials in advanced optoelectronic and photonic applications, including photovoltaics, due to its high transparency across the solar spectrum, excellent electrical properties, and the possibility to synthesize a rich variety of nanostructures ${ }^{9-12}$. Its abundance and non-toxicity are important additional criteria in view of the global large-scale deployment of photovoltaics. Approaches that have already been successfully employed to increase light trapping in solar modules on millions of square metres (see, for example, real-time solar panel counter at www.oerlikon.com/solar) include the growth of $\mathrm{ZnO}$ films with randomly oriented pyramids by means of chemical vapour deposition ${ }^{13}$ and wet etching of crater-like structures into sputtered $\mathrm{ZnO}$ films ${ }^{14}$. The pyramidal morphology in particular has demonstrated outstanding light-trapping capabilities and has led to several certified world-record conversion efficiencies ${ }^{15,16}$. Solution-based methods have also been extensively investigated for the synthesis of nanopillar-type $\mathrm{ZnO}$ structures ${ }^{9}$. Although all these approaches provide a certain degree of freedom in designing the surface morphology of $\mathrm{ZnO}$ films, the basic feature morphology (pyramids, craters or pillars) is dictated by the underlying growth and etch kinetics. Nanomoulding completely frees $\mathrm{ZnO}$ films from morphological constraints imposed by nature, and allows one to transfer or replicate an arbitrary master structure made from an arbitrary (transparent or opaque) master material onto a transparent $\mathrm{ZnO}$ electrode (Fig. 1). Consequently, nanomoulding provides full flexibility in designing light-trapping structures, moving one step further towards the fundamental limits for photonic light trapping predicted by theory ${ }^{17,18}$. We also expect that our nanomoulding technique may be extended to other materials and deposition methods, further widening its range of applications.
A sketch of the nanomoulding process flow is shown in Fig. 2. Starting from an arbitrary master structure (Fig. 2a), a negative mould is fabricated by nanoimprinting of the master texture into an ultraviolet (UV) curable sol-gel lacquer (Fig. 2b). After curing under UV light (Fig. 2c) and release from the master, the flexible mould is coated with an anti-sticking layer (Fig. 2d). Steps b to d are identical to the stamp fabrication steps for our recently developed high-fidelity UV nanoimprint lithography (UV-NIL) technique ${ }^{19}$. $\mathrm{ZnO}$ is subsequently grown on the mould by means of low-pressure chemical vapour deposition (LP-CVD) (Fig. 2e). The backside of the $\mathrm{ZnO}$ film is then anchored on a glass substrate using the UV-curable lacquer as a transparent glue (Fig. 2f). After
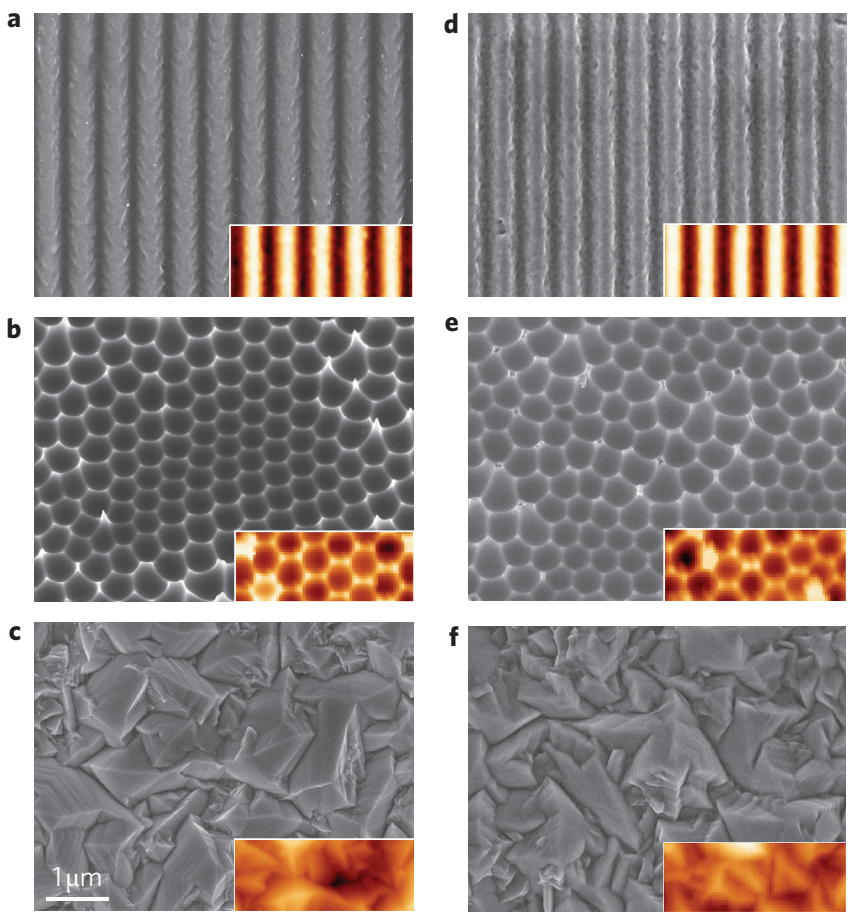

Figure 1 | SEM images of master test structures and their corresponding nanomoulded $\mathrm{ZnO}$ replicas. a, A one-dimensional periodic grating fabricated by interference lithography. b, A nanotextured aluminium surface with a quasi-periodic hexagonal dimple pattern prepared via anodic oxidation of aluminium and a subsequent etch-off of the aluminium oxide layer ${ }^{7,25}$. c, Random pyramid network of $\mathrm{ZnO}$ grown by low-pressure chemical vapour deposition. $\mathbf{d}-\mathbf{f}$, Images of the corresponding nanomoulded $\mathrm{ZnO}$ replicas Insets: AFM images. Image size, $6 \mu \mathrm{m} \times 4 \mu \mathrm{m}$. 

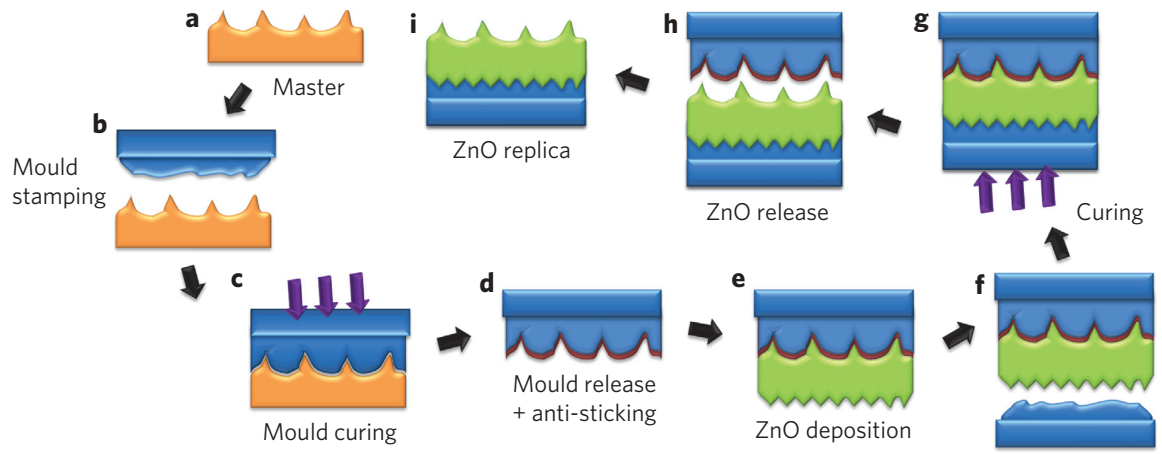

Anchoring

Figure 2 | Schematic process flow for nanomoulding of ZnO. a-i, The process can be divided into mould fabrication (b-d), ZnO deposition (e) and layer transfer $(\mathbf{f}-\mathbf{i})$. For details, see text.

curing under UV light (Fig. 2g) and detaching from the mould (Fig. 2h), a $\mathrm{ZnO}$ film is obtained that demonstrates a high-fidelity replication of the surface morphology of the master (Fig. 2i).

Figure 1a-c presents scanning electron microscopy (SEM) and atomic force microscopy (AFM) images of three different master test structures for nanomoulding. The corresponding $\mathrm{ZnO}$ replicas are shown in Fig. 1d-f. The reproduction fidelity of the dominant morphological features (grating lines, dimples and pyramids) is striking. Even fine details, much smaller than those relevant to visible and near-infrared light scattering, have been reproduced with excellent accuracy, as can be seen in the SEM images. Small modulations along the grating rims of the master in Fig. 1a can still be seen in the replica (Fig. 1d). The onsets of the sharp spikes, occurring at domain boundaries in Fig. 1b, are also clearly distinguished on the replica in Fig. 1e, although the spikes are not replicated up to the tip. Even fine crystal dislocation lines on the faces of the $\mathrm{ZnO}$ pyramids in Fig. 1c are still visible on the replica in Fig. 1f.

For a quantitative assessment of the replication quality, we extracted local height and angle histograms from the AFM images. From inspection of Fig. $3 a-c$, it can be seen that the height histograms of the master and $\mathrm{ZnO}$ replica are very similar for all structures. Focusing now on the angle histograms in Fig. $3 \mathrm{~d}-\mathrm{f}$, which are very sensitive to subtle morphological changes, it is apparent that the replicated structures have only a slight tendency to smaller angles. The highest angle fidelity is observed for the replica of the pyramidal $\mathrm{ZnO}$ structure, possibly because of the genuine $\mathrm{ZnO}$ angle distribution of the mould. Further studies are necessary to assess the resolution limit for nanomoulding. The ultimate resolution will not only depend on mould precision, but also on $\mathrm{ZnO}$ growth parameters.

To assess the optical scattering properties of nanomoulded structures, we focus now on the pyramidal $\mathrm{ZnO}$ structure. Both the $\mathrm{ZnO}$ master and $\mathrm{ZnO}$ replica are transparent and made of the same material, allowing direct comparison of their optical properties. Angle (Fig. 4a) and wavelength (Fig. 4b) dependence of the scattered light almost coincide for the master and $\mathrm{ZnO}$ replica. Figure 4 also shows the scattering characteristics of the nanomoulded grating and the nanomoulded dimple structure of anodically textured aluminium (ATA), demonstrating that very different scattering behaviour can be obtained by engineering the $\mathrm{ZnO}$ electrode morphology.

For optoelectronic applications it is of prime importance that the electrical properties of the transferred nanomoulded $\mathrm{ZnO}$ film are conserved. To verify this, we determined the electron mobility by means of Hall measurements and found $45 \mathrm{~cm}^{2} \mathrm{~V}^{-1} \mathrm{~s}^{-1}$ for the film deposited on the pyramidal mould and transferred to the glass substrate. For a layer co-deposited on a flat glass substrate, we found a very similar value of $47 \mathrm{~cm}^{2} \mathrm{~V}^{-1} \mathrm{~s}^{-1}$. Thus, the excellent electrical properties are fully conserved during the layer transfer.
We then deposited an amorphous silicon solar cell on the pyramidal $\mathrm{ZnO}$ replica and compared it to a cell on the master $\mathrm{ZnO}$ film grown directly on glass. Figure 5 presents SEM images of cross-sections through the two solar cells milled with a focused ion beam (FIB). Careful comparison of the grain structure of the $\mathrm{ZnO}$ front electrodes clearly reveals the reversed grain configuration of the moulded $\mathrm{ZnO}$ front contact in Fig. 5b, with the $\mathrm{ZnO}$ nucleation layer adjacent to the active silicon layers. It is exactly the fine-grained nature of this nucleation layer that is responsible for the high fidelity of the nanomoulding process.

Figure $5 \mathrm{~d}$ presents external quantum efficiency (EQE) measurements for these cells. For comparison, we also show the EQE measurement for a cell grown on a flat $\mathrm{ZnO}$ front electrode, with no light trapping. The effect of light trapping is manifested as a
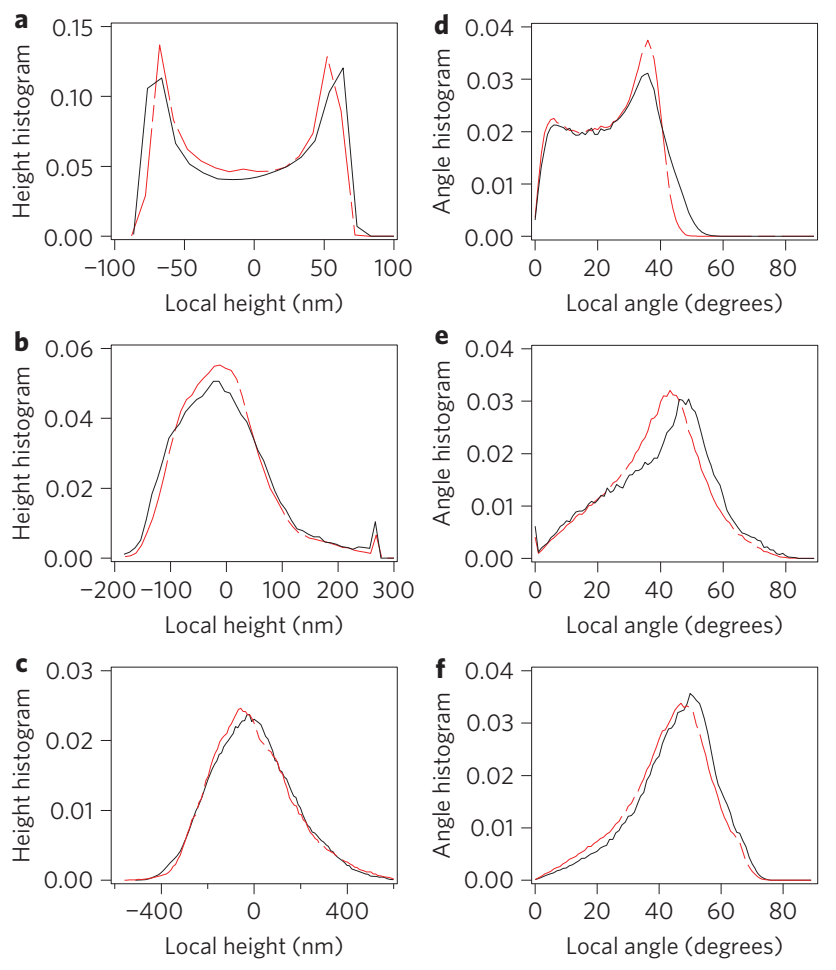

Figure 3 | Morphological fidelity analysis for $\mathrm{ZnO}$ nanomoulding. a-f, Local height (a-c) and angle (d-f) histograms extracted from the AFM images of the three test structures in Fig. 1. Master and replica histograms are shown as black solid and red dashed lines, respectively. Bin widths are $10 \mathrm{~nm}$ and $1^{\circ}$, respectively, and the area under the histogram is normalized to 1. 

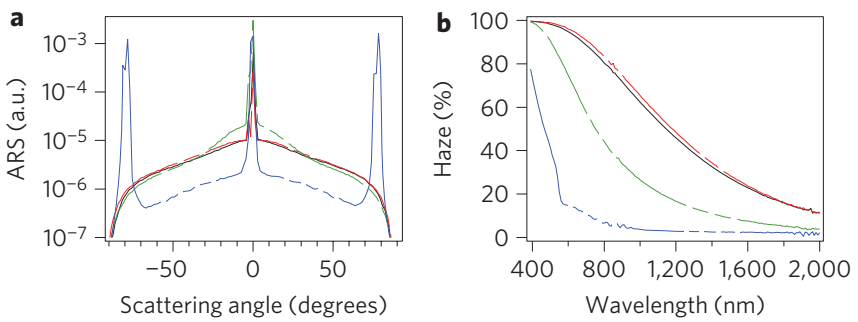

Figure 4 | Comparison of optical scattering properties. a, Angle resolved scattering (ARS) intensity at a wavelength of $543 \mathrm{~nm}$. b, Ratio between diffuse and total transmittance (haze) as a function of wavelength for the pyramidal $\mathrm{ZnO}$ master (black line), its $\mathrm{ZnO}$ replica (red line), the ATA replica (green line) and the grating replica (blue line). a.u., arbitrary units.
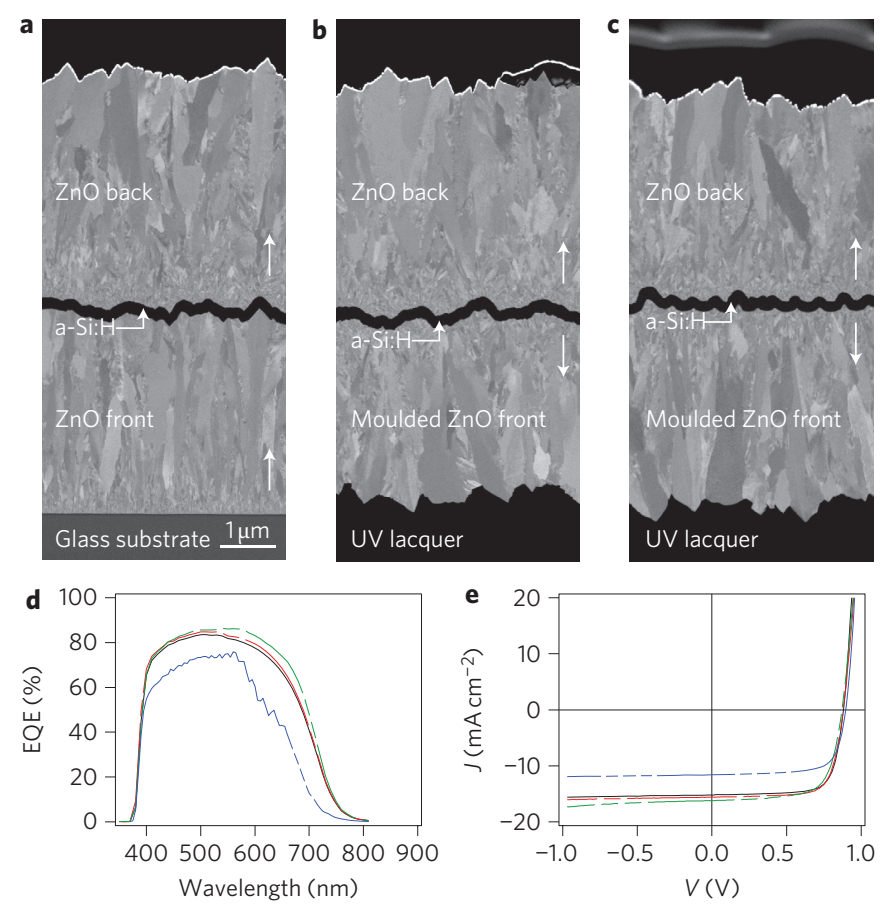

Figure 5 | Amorphous silicon solar cells on nanomoulded ZnO. a-c, FIB cuts across the solar cells on the master $\mathrm{ZnO}$ (a), the nanomoulded $\mathrm{ZnO}$ replica (b), and the nanomoulded ATA replica (c). Growth direction of the $\mathrm{ZnO}$ layers is indicated by white arrows. d,e, EQE (d) and $J(V)(\mathbf{e})$ characteristics of the cells on the pyramidal $\mathrm{ZnO}$ master (black line), its $\mathrm{ZnO}$ replica (red line) and the ATA replica (green line). The EQE and $J(V)$ characteristics of a cell deposited on flat $\mathrm{ZnO}$ (blue line), without light trapping, are also shown. The thickness of the intrinsic amorphous silicon layer is $250 \mathrm{~nm}$.

massive conversion enhancement, boosting the photo-generated current by more than $30 \%$ when nanotextured interfaces are introduced (see Table 1 for a summary of cell parameters). The EQE curves for the $\mathrm{ZnO}$ master and nanomoulded $\mathrm{ZnO}$ replica are almost identical. Figure $5 \mathrm{c}$,d also presents a FIB cut and the EQE data of a cell deposited on the nanomoulded ATA structure from Fig. 1e. This cell illustrates the use of nanomoulding to transfer textures from opaque masters to transparent electrodes. In terms of short-circuit current density $J_{\mathrm{sc}}$ (given in Table 1), the ATA structure outperforms the $\mathrm{ZnO}$ structure by $0.6 \mathrm{~mA} \mathrm{~cm}^{-2}$, demonstrating that substantial further improvements in light trapping are achievable by integrating advanced photonic nanostructures.

Cell parameters, extracted from the current density versus voltage $(J(V))$ characteristics shown in Fig. 5e, are summarized in
Table 1 | Characteristics of amorphous silicon solar cells grown on the $\mathrm{ZnO}$ master, its $\mathrm{ZnO}$ replica, flat $\mathrm{ZnO}$ and replicated ATA.

\begin{tabular}{lllll} 
& $\boldsymbol{J}_{\mathbf{s c}}\left(\mathrm{mA} \mathrm{cm}^{-\mathbf{2}}\right)$ & $\boldsymbol{V}_{\text {oc }}(\mathbf{m V})$ & $\mathbf{F F}(\%)$ & Efficiency (\%) \\
\hline ZnO master & 15.2 & 880 & 76 & 10.1 \\
ZnO replica & 15.6 & 879 & 74 & 10.1 \\
ZnO flat & 11.6 & 896 & 73 & 7.6 \\
ATA replica & 16.2 & 871 & 70 & 9.9
\end{tabular}

Table 1. The cell on the $\mathrm{ZnO}$ replica exhibits an excellent initial efficiency of $10.1 \%$, a value as high as for the cell on the state-ofthe-art master $\mathrm{ZnO}$. Although the ATA morphology results in a higher $J_{\text {sc }}$, a slightly lower efficiency of $9.9 \%$ is obtained due to a reduced open-circuit voltage $V_{\text {oc }}$ and fill factor FF caused by morphology-induced undesirable local current drains in the active silicon layer acting as electrical shunts ${ }^{20}$. It remains one of the major challenges of thin-film solar cells to find an optimal morphology that maximizes light trapping while maintaining high electrical performance. Promising recent developments towards more tolerant silicon layers ${ }^{21,22}$, combined with nanomoulding, provide a clear roadmap for the integration of even more aggressive nanostructures into the cells.

We recently reported the use of UV nanoimprint lithography for the fabrication of transparent nanotextured substrates for solar cell applications ${ }^{8,23}$. Similar to nanomoulding, nanoimprinting allows the integration of a large variety of nanostructures into cells. Nanoimprinting is performed into a deformable insulating sol-gel lacquer, and requires an additional transparent conductive layer to operate as the front electrode. Although $\mathrm{ZnO}$ is non-deformable and brittle, rendering nanoimprinting of $\mathrm{ZnO}$ impossible, nanomoulding offers an elegant alternative method with the additional advantage that the structure is directly fabricated out of a transparent conducting material.

An important feature of $\mathrm{ZnO}$ nanomoulding is its compatibility with industrial in-line mass production. Analogous to nanoimprinting techniques ${ }^{24}$, nanomoulding is directly upscalable to large-area modules. $\mathrm{ZnO}$ deposition on the mould and the subsequent transfer onto glass substrates may be repeated many times using the same mould. Nanomoulding is also fully compatible with monolithic series interconnection of cell segments to form full modules by means of laser scribing. Moreover, we were not able to observe any significant degradation of the optical properties of the anchoring lacquer under prolonged UV light and humidity exposure, which is important for the long-term stability of solar modules.

In conclusion, we have developed a new versatile technology enabling precise large-area nanopatterning of transparent $\mathrm{ZnO}$ electrodes at low cost. We achieved conversion efficiencies for cells deposited on nanomoulded electrodes as high as those on state-ofthe-art nanotextured electrodes, proving that $\mathrm{ZnO}$ nanomoulding allows one to go far beyond proof-of-concept devices. Our approach holds promise for significant further efficiency improvements, not only by careful device optimization, but also by providing a general tool for exploring the light-trapping performance of specifically designed photonic nanostructures directly in high-efficiency solar cells and modules. We anticipate a strong potential for other thin-film photovoltaic technologies, including cadmium telluride $(\mathrm{CdTe})$, copper indium gallium diselenide (CIGS) and organic and dye-sensitized solar cells, as well as for other optoelectronic and photonic applications.

\section{Methods}

Mould fabrication and ZnO layer transfer. For mould fabrication (Fig. 2b) and anchoring of the $\mathrm{ZnO}$ film (Fig. 2f), a UV-curable sol-gel lacquer (Ormocer from Micro Resist Technology GmbH) was spin-coated on a flexible 0.125-mm-thick polyethylene naphthalate (PEN) sheet and a $0.5-\mathrm{mm}$-thick borosilicate glass substrate (Schott AF45) with dimensions of $41 \mathrm{~mm} \times 41 \mathrm{~mm}$ without antireflection 
coating. Stamping and anchoring was performed in a home-built UV-NIL system under vacuum, to avoid bubble formation, by applying a homogeneous pressure of 1 bar. After curing under UV light (Fig. 2c,g), release (Fig. 2d,h) was performed manually. Before $\mathrm{ZnO}$ deposition, the flexible mould was coated with a 10-nm-thick sputtered chromium layer and an anti-adhesion monolayer of trichloro(tridecafluorooctyl)silane (Fig. 2d). The grating mould was fabricated from a UV-NIL replica ${ }^{19}$ of the grating master (GH50-18V from Thorlabs). No chromium layer was used for the grating. The ATA master was fabricated by anodic oxidation of aluminium (Woo Lee, Korea Institute of Standards and Science). The ZnO master was deposited as described below.

$\mathrm{ZnO}$ deposition. $\mathrm{ZnO}$ films were grown on the mould using LP-CVD at $180{ }^{\circ} \mathrm{C}$ (Fig. 2e) using $\left(\mathrm{C}_{2} \mathrm{H}_{5}\right)_{2} \mathrm{Zn}$ and $\mathrm{H}_{2} \mathrm{O}$ as precursor gases. Doping was achieved by dosing small amounts of $\mathrm{B}_{2} \mathrm{H}_{6}$ diluted at $1 \%$ in argon into the gas mixture. Flat $\mathrm{ZnO}$ electrodes were fabricated by chemical-mechanical polishing of as-grown pyramidal $\mathrm{ZnO}$ films.

$\mathrm{ZnO}$ characterization. The morphology of the moulded $\mathrm{ZnO}$ surfaces was characterized by SEM (JEOL JSM-7500 TFE) and AFM (Digital Instruments Nanoscope 3100). The SEM images in Fig. 1 were taken with master and corresponding replica located side by side on the same sample holder without changing the electron beam and detector parameters. The chemical sensitivity of SEM clearly results in contrast and brightness differences between the images. A certain difference in SEM contrast is also seen between master and replica in Fig. $1 \mathrm{c}$ and $\mathrm{f}$, although both are made of $\mathrm{ZnO}$. We attribute this to the fact that for the master in Fig. 1c the sharp edges of single-crystalline grains lead to a strong contrast, whereas it is the nucleation layer that is facing the surface on the replica in Fig. If, resulting in less contrast. Angle-resolved scattering of light was measured with a silicon detector on a home-built goniometer under normal incidence onto the glass side using a laser at a wavelength of $543 \mathrm{~nm}$. The spectral dependence of total and diffuse transmittance was determined with a photospectrometer (Perkin Elmer Lambda 900) equipped with an integrating sphere. Hall measurements were carried out in the Van Der Pauw configuration.

Solar cell deposition. Amorphous silicon solar cells with an intrinsic layer thickness of $250 \mathrm{~nm}$ were deposited by plasma-enhanced CVD in an industrial KAI reactor with parallel plate configuration at $200{ }^{\circ} \mathrm{C}$. $\mathrm{SiH}_{4}$ and $\mathrm{H}_{2}$ were used as source gases for intrinsic layers. For doped layers, we also used $\mathrm{CH}_{4}$ and $\mathrm{CO}_{2}$. p- and n-doping was achieved by adding $\mathrm{PH}_{3}$ and $\mathrm{B}\left(\mathrm{CH}_{3}\right)_{3}$, respectively. After cell deposition, a $\mathrm{ZnO}$ back contact was deposited as described above. The cells were patterned to $5 \times 5 \mathrm{~mm}^{2}$ by lift-off and $\mathrm{SF}_{6}$ reactive ion etching.

Solar cell characterization. Cross-sections across the solar cells were milled and imaged by FIB-SEM (Zeiss NVision 40 Crossbeam). Open-circuit voltage $\left(V_{\mathrm{oc}}\right)$ and fill factor (FF) were calculated from the current density-voltage $(J(V))$ characteristics of the cells, measured with a dual lamp sun simulator in standard test conditions $\left(25^{\circ} \mathrm{C}\right.$, global air mass 1.5 (AM1.5 $)$ spectrum, $1,000 \mathrm{~W} \mathrm{~m}^{-2}$ ). The short-current density $\left(J_{\mathrm{sc}}\right)$ was determined by convolution of the EQE and the incoming photon flux of the AM1.5 $\mathrm{g}$ spectrum.

Received 9 March 2011; accepted 15 July 2011; published online 21 August 2011

\section{References}

1. Zhu, J., Hsu, C-M., Yu, Z., Fan, S. \& Cui, Y. Nanodome solar cells with efficient light management and self-cleaning. Nano Lett. 10, 1979-1984 (2010).

2. Garnett, E. \& Yang, P. Light trapping in silicon nanowire solar cells. Nano Lett. 10, 1082-1087 (2010).

3. Kelzenberg, M. D. et al. Enhanced absorption and carrier collection in Si wire arrays for photovoltaic applications. Nature Mater. 9, 239-244 (2010).

4. Cao, L. et al. Semiconductor nanowire optical antenna solar absorbers. Nano Lett. 10, 439-445 (2010).

5. Han, S. E. \& Chen, G. Optical absorption enhancement in silicon nanohole arrays for solar photovoltaics. Nano Lett. 10, 1012-1015 (2010).

6. Atwater, H. A. \& Polman, A. Plasmonics for improved photovoltaic devices. Nature Mater. 9, 205-213 (2010).

7. Sai, H., Fujiwara, H., Kondo, M. \& Kanamori, Y. Enhancement of light trapping in thin-film hydrogenated microcrystalline Si solar cells using back reflectors with self-ordered dimple pattern. Appl. Phys. Lett. 93, 143501 (2008).
8. Battaglia, C. et al. Nanoimprint lithography for high-efficiency thin-film silicon solar cells. Nano Lett. 11, 661-665 (2010).

9. Lincot, D. Solution growth of functional zinc oxide films and nanostructures. MRS Bull. 35, 778-789 (2010).

10. Özgür, U. et al. A comprehensive review of $\mathrm{ZnO}$ materials and devices. J. Appl. Phys. 98, 041301 (2005).

11. Tian, Z. R. et al. Complex and oriented $\mathrm{ZnO}$ nanostructures. Nature Mater. 2, 821-826 (2003).

12. Law, M., Greene, L. E., Johnson, J. C., Saykally, R. \& Yang, P. Nanowire dyesensitized solar cells. Nature Mater. 4, 455-459 (2005).

13. Faÿ, S., Steinhauser, J., Oliveira, N., Vallat-Sauvain, E. \& Ballif, C. Optoelectronic properties of rough LP-CVD $\mathrm{ZnO}: \mathrm{B}$ for use as TCO in thin-film silicon solar cells. Thin Solid Films 515, 8558-8561 (2007).

14. Berginski, M., Hüpkes, J., Reetz, W., Rech, B. \& Wuttig, M. Recent development on surface-textured $\mathrm{ZnO}$ :Al films prepared by sputtering for thin-film solar cell application. Thin Solid Films 516, 5836-5841 (2008).

15. Benagli, S. et al. High-efficiency amorphous silicon devices on LPCVD-ZnO TCO prepared in industrial KAI-M R\&D reactor. Proceedings of the 24th European Photovoltaic Solar Energy Conference, Hamburg, Germany, 2293-2298 (2009).

16. Bailat, J. et al. Recent developments of high-efficiency micro-morph tandem solar cells in KAI-M PECVD reactors. Proceedings of the 5th World Conference on Photovoltaic Energy Conversion, Valencia, Spain, 2720-2723 (2010).

17. Yablonovitch, E. Statistical ray optics. J. Opt. Soc. Am. 72, 899-907 (1982).

18. Yu, Z., Raman, A. \& Fan, S. Fundamental limit of nanophotonic light trapping in solar cells PNAS. Proc. Natl Acad. Sci. USA 107, 17491-17496 (2010).

19. Escarré, J., Söderström, K., Haug, F-J., Battaglia, C. \& Ballif, C. High fidelity transfer of nanometric random textures by UV embossing for thin film solar cells applications. Sol. Energ. Mater. Sol. Cells. 95, 881-886 (2011).

20. Python, M. et al. Influence of the substrate geometrical parameters on microcrystalline silicon growth for thin-film solar cells. Sol. Energ. Mater. Sol. Cell. 93, 1714-1720 (2009).

21. Despeisse, M. et al. Resistive interlayer for improved performance of thin film silicon solar cells on highly textured substrate. Appl. Phys. Lett. 96, 073507 (2010)

22. Cuony, P. et al. Mixed-phase p-type silicon oxide containing silicon nanocrystals and its role in thin-film silicon solar cells. Appl. Phys. Lett. 97, 213502 (2010)

23. Battaglia, C. et al. Efficient light management scheme for thin film silicon solar cells via transparent random nanostructures fabricated by nanoimprinting. Appl. Phys. Lett. 96, 213504 (2010).

24. Ahn, S. H. \& Guo, L. J. Large-area roll-to-roll and roll-to-plate nanoimprint lithography: a step toward high-throughput application of continuous nanoimprinting. ACS Nano 3, 2304-2310 (2009).

25. Lee, W., Ji, R., Gösele, U. \& Nielsch, K. Fast fabrication of long-range ordered porous alumina membranes by hard anodization. Nature Mater. 5, 741-747 (2006)

\section{Acknowledgements}

The authors thank D. Alexander and M. Leboeuf for assistance with the FIB and AFM, respectively, W. Lee for providing the ATA master, Z. Holman and P. Cuony for careful proof-reading of the manuscript, and the Swiss Federal Energy Office and the Swiss National Science Foundation for funding (under project no. 101191 and grant no. $20002112577 / 1)$

\section{Author contributions}

C.B. conceived the nanomoulding method and coordinated the work. J.E. carried out the first nanomoulding experiments. K.S. fabricated the nanomoulded grating. M.C. deposited the silicon layers. C.B. characterized the nanomoulded $\mathrm{ZnO}$, processed and measured the cells, performed the data analysis and wrote the manuscript. M.D. led the silicon layer development. F.-J.H. led the nanoimprinting activities. Ch.B. supervised the work.

\section{Additional information}

The authors declare no competing financial interests. Reprints and permission information is available online at http://www.nature.com/reprints. Correspondence and requests for materials should be addressed to C.B. 